\title{
Implantación de una Unidad de Ortogeriatría en un hospital de tercer nivel
}

\author{
$\mathrm{M}^{\mathrm{a}}$ de los Ángeles Cruz ${ }^{1}$ y Manuel Lillo ${ }^{2}$ \\ ${ }^{1}$ Hospital Universitario Nuestra Señora de Candelaria, Tenerife (España); \\ ${ }^{2}$ Universidad de Alicante (España)
}

El envejecimiento de la población española ha llevado a que cada vez sea mayor el número de fracturas de cadera que son atendidas. Son pacientes que por su fragilidad, necesitan una atención especial, siendo las Unidades de Ortogeriatría (UOG), una de las que más beneficios han demostrado. El objetivo principal de este proyecto es evaluar las principales variables, de los pacientes ancianos con fracturas de cadera, atendidas en una UOG de nueva creación, en el Hospital Universitario Nuestra Señora de Candelaria. Se realiza un estudio descriptivo observacional de los pacientes ancianos con fractura de cadera ingresados en una planta convencional de Traumatología, y cuáles de ellos son derivados a UOG. Se registró: el tiempo de ingreso, el tipo de fractura e intervención, las sesiones de fisioterapia en hospitalización convencional, en la UOG, en la Unidad de Hospitalización a domicilio (HADO) y cuántos de ellos han necesitado continuar tratamiento de fisioterapia ambulatoria. El inicio precoz de la fisioterapia en hospitalización, que se completa en la UOG y/o el ingreso en HADO, junto con el asesoramiento a las familias y a los pacientes, ha demostrado reducir en un porcentaje muy elevado la necesidad de continuar la rehabilitación ambulatoria.

Palabras clave: Fractura de cadera, anciano, fisioterapia, Unidad Ortogeriátrica.

Start-up of an orthogeriatric unit in a specialized general hospital. The aging of the Spanish population has led to an increasingly greater number of hip fractures attended by the Spanish Healthcare System in the last years. Those patients, due to their fragility, need special professional attention and consequently, Orthogeriatric Units (UOG) have achieved the highest benefits. The main purpose of this research project is to evaluate the principle variables associated to the elderly affected of hip fracture staying in a new Orthogeriatric Unit created at the Hospital Nuestra Señora de Candelaria in Tenerife, Spain. A descriptive study of the elderly patients diagnosed of hip fracture staying in a conventional Orthopedic Ward was conducted, focusing specifically in those who were transferred to the UOG. The main items to describe were: total time of in-hospital staying, type of hip fracture and the kind of surgery selected, physiotherapist sessions in the conventional Orthopedic Ward, in the UOG and in Home Hospitalization Unit (HADO) as well as the total number of patients that needed to continue ambulatory physiotherapist sessions. As a conclusion, early start of physiotherapist sessions inside the hospital, completed in the UOG and/or in HADO, along with counseling and professional assessment to families and patients, reduced a very high percentage of their need for ambulatory ongoing rehabilitation.

Keywords: Hip fracture, elderly, physiotherapy, Orthogeriatric Unit.

$\overline{\text { Correspondencia: } \mathrm{M}^{\mathrm{a}}}$ de los Ángeles Cruz Marrero. Hospital Universitario Nuestra Señora de Candelaria. Ctra. General del Rosario, 145. C.P.: 38010. Santa Cruz de Tenerife (España). E-mail: angelescruz67@hotmail.com 
La fractura de cadera es una de las causas principales de morbilidad y mortalidad en personas mayores, lo que significa una gran repercusión en la sociedad. Aproximadamente el $85 \%$ de todas las fracturas de cadera se producen en personas mayores de 65 años (SEGG, 2007).

En los países desarrollados, el tratamiento de una fractura de cadera requiere un abordaje multidisciplinar (Parker, 2006), ya que el paciente llega al servicio de urgencias, a continuación, pasa a través de los departamentos de radiología, anestesia, cirugía ortopédica, medicina y rehabilitación, y se pueden necesitar los servicios médicos y sociales en la comunidad cuando el paciente abandona el hospital.

A finales del año 2011, según nuestro Instituto Nacional de Estadística (INE, 2012), un 23.2\% de la población era anciana, es decir, tenía más de 64 años. Más en concreto, un 5.6\% tenía más de 79 años, observándose en este grupo etario un incremento mantenido de su población en la última década.

En esta población que tiene una mayor esperanza de vida las enfermedades crónicas son las que provocan un mayor gasto sanitario, y si a ello añadimos la complicación de una fractura de cadera el gasto se dispara. A medida que avanza la edad, disminuye el porcentaje de personas que no necesitan ayuda para realizar determinadas tareas, como en el grupo de 85 y más años, en el que solo un $22 \%$ de varones y un $11.4 \%$ de mujeres declaran que pueden realizar por sí mismas una serie de tareas (INE, 2012).

La dependencia funcional más frecuente en hombres de 65 y más años es para las tareas domésticas $(30.6 \%)$, mientras que en mujeres la más frecuente es para la movilidad (43.7\%) (INE 2012).

Esta fractura es una lesión grave y ocurre con frecuencia en mayores frágiles, generalmente como resultado de una caída simple desde su propia altura, según se publica la Sociedad Española de Geriatría y Gerontología (SEGG, 2007). Teniendo en cuenta que se produce en persona que en su mayoría tienen patologías concomitantes, problemas de deterioro cognitivo o demencia, y en la mayoría de los casos osteoporosis, la incidencia de las fracturas osteoporóticas en España es mucho mayor de la que se suponía, 720 casos anuales por cada 100.000 mayores de 60 años (Ganeglius, 2011; Sánchez-Lacuesta, 2005) cifra superior a la publicada por Serra (2002). La mayor incidencia se produce en los grupos de edad de más de 74 años, que es cuatro veces mayor que la incidencia total (915.4 por 100.000), esto puede contribuir a su muerte, la pérdida de su independencia y de su modo de vida previo a la factura de cadera, factores que se han tenido en cuenta en la elaboración de en el Plan de Acción desarrollado en la Comisión Europea sobre la Osteoporosis, para conseguir una Europa sin fracturas por fragilidad (2003) y (Hernlund, 2013).

El número de hospitalizaciones (altas) por fractura de cadera que se han producido en nuestro país aumentado de forma continua desde el año 1997 hasta 2012, 
con la salvedad del año 1999 en el que se registró un ligero descenso en relación con el periodo anterior, según la publicación del Ministerio del Interior realizada por el Instituto de Información Sanitaria. Estadísticas Comentadas: La Atención a la Fractura de Cadera en los Hospitales del SNS (2010). En términos absolutos, estas altas pasaron de 34.876 a 66.049, de las cuales 18.271 eran hombres y 47.778 mujeres (INE, 2012).

En nuestro trabajo nos vamos a centrar, en las facturas de cuello de fémur, clasificadas con los código CMBD (820 y 821), que se han presentado en la Comunicad Canaria, más concretamente en la Provincia de Santa Cruz de Tenerife, tomando como referencia las Encuestas de morbilidad realizadas por el INE durante el año 2012, hemos desarrollado esta tabla.

Tabla 1. Encuestas de morbilidad

\begin{tabular}{|c|c|c|c|}
\hline & TOTAL NACIONAL & CANARIAS & Santa Cruz de Tenerife \\
\hline \multicolumn{4}{|l|}{ Ambos sexos } \\
\hline $\begin{array}{l}\text { 820-821 Fracturas del cuello de fémur, de } \\
\text { otras partes y de partes no especificadas }\end{array}$ & 66.049 & 2.008 & 1.183 \\
\hline \multicolumn{4}{|l|}{ Hombres } \\
\hline $\begin{array}{l}\text { 820-821 Fracturas del cuello de fémur, de } \\
\text { otras partes y de partes no especificadas }\end{array}$ & 18.271 & 648 & 334 \\
\hline \multicolumn{4}{|l|}{ Mujeres } \\
\hline $\begin{array}{l}\text { 820-821 Fracturas del cuello de fémur, de } \\
\text { otras partes y de partes no especificadas }\end{array}$ & 47.778 & 1.360 & 849 \\
\hline
\end{tabular}

En la Unión Europea se calcula que el coste de la fractura osteoporótica alcanza los 25 millones de euros, además hay que considerar los gastos indirectos (estancias en residencias, cuidadores), que aumentan considerablemente esta cifra, a lo que hay que añadir el cuidado del paciente al año siguiente de sufrir la fractura (GEIOS, 2011).

En la actualidad en España se estima entre 8.000-9.000 fracturas ingresadas y operadas con diferente complejidad con unos costes promedios 35 millones de euros para las fracturas ingresadas y otros 10 millones para las no ingresadas (GEIOS, 2011).

Costes por cada fractura $4.000 €$ a $11.000 €$ por paciente.

Costes totales anuales alrededor 300 millones de $€$.

De las personas que sufren fractura de cadera ente el 12 y $37 \%$ muere durante el primer año y entre el 10 y $20 \%$ requiere un cambio de estado residencial (Pidemunt, 2009).

Esta situación que es generalizada en todos los países desarrollados ha provocado el estudio y decisión de la necesidad del trabajo multidisciplinar, que conlleva la atención de este tipo de paciente por ello se han desarrollado tanto a nivel nacional como internacional diversas Guías Clínicas sobre los pacientes con fractura de cadera ancianos, donde se definen y protocolizan los diferentes profesionales que intervienen en el proceso desde que el paciente llega el servicio de urgencias y las secuencias más 
adecuadas en las que actúan cada uno de ellos de forma protocolizada (enfermera, médico de urgencia, traumatólogo, anestesista, internista, médico rehabilitador, fisioterapeuta...) (National Clinical Guideline Centre, 2011; Misterio de Sanidad y Política Social, 2010; Guías Clínicas de la Sociedad Gallega de Medicina Interna, 2005; Hospital de Donostia Protocolo fractura cadera, 2007; Hospital La Fuentefría Comunidad de Madrid, 2005; Protocolo de Actuación en las Fracturas de Cadera en el anciano en el Hospital General Universitario de Alicante, 2007; Olias de Lima, 2012).

Los efectos de la rehabilitación multidisciplinar en contextos hospitalario, ambulatorio o en domicilio en pacientes mayores con fractura de cadera son de vital importancia, ya que afecta directamente a la reducción de las estancias hospitalarias, y a los costes de la carga para los cuidadores (Handoll, Sherrington y Parker, 2004; Handoll y Sherrington, 2007; Hoeing, Sanford, Butterfield, Griffiths y Hargraves, 2006; National Clinical Guideline Centre, 2011).

Si bien ha habido un trabajo limitado en la aplicación del ejercicio de actividades posteriores a la fractura de cadera, hay algún tipo de apoyo que sugiere importantes beneficios para estas personas, hay múltiples trabajos y revisiones de trabajos publicados (Hoeing et al., 2006; Liu y Latham, 2009), sobre los tipos de rehabilitación, intensiva y con programas de alta resistencia (Moseley, Sherrington, Lord, Barraclough, St George y Cameron, 2009; Tinetti, Baker, Gottschalk y Williams, 1999), realizada en centros específicos de rehabilitación (Crotty, Enroje, Cameron, Miller, Ramírez y Couzner, 2010), en domicilio (Binder, Brown, Sinacore, Steger-May, Yarasheski y Schechtman, 2004; Liu y Latham, 2009; Mangione, Craik, Tomlinson y Palombaro, 2005), y los beneficios de dichos tratamientos a corto y largo plazo. En concreto, para aquellos que han sufrido una fractura de cadera, el ejercicio regular (resistente y/o aeróbica) mejora la movilidad y la fuerza del cuádriceps, aumenta la velocidad al caminar y la capacidad de soporte de peso (Barker, Dewey y Sackley, 2009; Crotty, 2010).

No hay suficientes pruebas a partir de los ensayos aleatorios como para determinar los efectos de cualquier estrategia o programa de movilización particular que comenzó en el periodo de rehabilitación temprano o tardío después de la cirugía por fractura de cadera. Sin embargo, los ensayos incluidos indican generalmente que es posible mejorar la movilidad después de una fractura de cadera, aunque el método óptimo para lograrlo no está claro. Aunque los programas más exitosos evaluados hasta la fecha incluyeron ejercicios continuos intensivos supervisados, no se han establecido el formato óptimo y las implicaciones de recursos para estas estrategias, la Fisioterapia ha sido un componente rutinario de la rehabilitación de los pacientes después del reemplazo de articulación de la cadera (Barker et al., 2009; Crotty et al., 2010; Liu y Latham, 2009; Minns et al., 2009; Moseley et al., 2009). 
El éxito de estas Unidades de ortogeriatría se debe al trabajo multidisciplinar planificado de un equipo que dialoga (Bermejo, 2013) donde cada profesional de la salud aporta de manera integral el tratamiento adecuado a los pacientes, deben formar parte de él: geriatra, rehabilitador, enfermeras, fisioterapeutas, terapeutas ocupacionales, asistente social y psicólogo. Los miembros del equipo exponen sus resultados, se discute, y se decide de forma coordinada el programa de cada paciente, se hace un seguimiento conjunto hasta el alta, y su integración en la comunidad en las mejores condiciones posibles (Bielza, Ortiz, Arias, Velasco, Mora, Moreno, Tapia, Escalera y Gómez, 2013).

La Unidad de Ortogeriatría del Hospital La Paz, que ha tratado a más de 4.500 pacientes desde su puesta en marcha en 2007, expone que permite acelerar la recuperación de los ancianos con fractura de cadera, disminuyendo las complicaciones y reduciendo el coste asistencial, lo que ha permitido diagnosticar a más pacientes reduciendo el tiempo desde el ingreso hasta la intervención y desde la intervención hasta el inicio de la fisioterapia (Europa Press, 2009).

"En las UOG, los pacientes ancianos con fracturas de cadera, no sólo consiguen recuperar su funcionalidad, sino disminuir la mortalidad hospitalaria, y todo ello en el menor tiempo y al menor coste posible" (Europa Press, 2009).

Todos estos antecedentes han promovido la creación de una Unidad de Ortogeriatría en el Hospital Universitario Nuestra Señora de la Candelaria en Santa Cruz de Tenerife. El objetivo de este estudio fue, evaluar las principales variables, de los pacientes con fracturas de cadera ancianas que han sido atendidos en esta Unidad desde su creación hasta Octubre 2013. De este objetivo nos planteamos la hipótesis:

El inicio precoz de la fisioterapia hospitalaria en la Unidad de Ortogeriatría, junto con el asesoramiento a las familias y los pacientes, reduce en un porcentaje muy elevado la necesidad de continuar la rehabilitación ambulatoria.

\section{MÉTODO}

Estudio descriptivo observacional con control del ingreso de pacientes ancianos con fractura de cadera ingresados en una planta convencional de Traumatología y cuáles de ellos son derivados a UOG.

\section{Participantes}

La muestra es de 424 pacientes que han sido intervenidos de fractura de cadera en el Hospital Nuestra Señora de la Candelaria en Santa Cruz de Tenerife, entre enero de 2012 y octubre de 2013.

De la muestra obtenida, los pacientes incluidos para este trabajo eran ancianos, es decir, pacientes de mayor o igual a 65 años. Aplicando dicho parámetro la muestra se queda en 376 pacientes. De los 376 pacientes, $75 \%$ (282) son mujeres y el 
$25 \%$ (94) son hombres. La edad media de los pacientes es de $82 \pm 7$ años. Sin diferencia significativas con $p=0.127$ (Figura 1).

Figura 1. Edad media de la muestra, de pacientes ingresados en U.O.

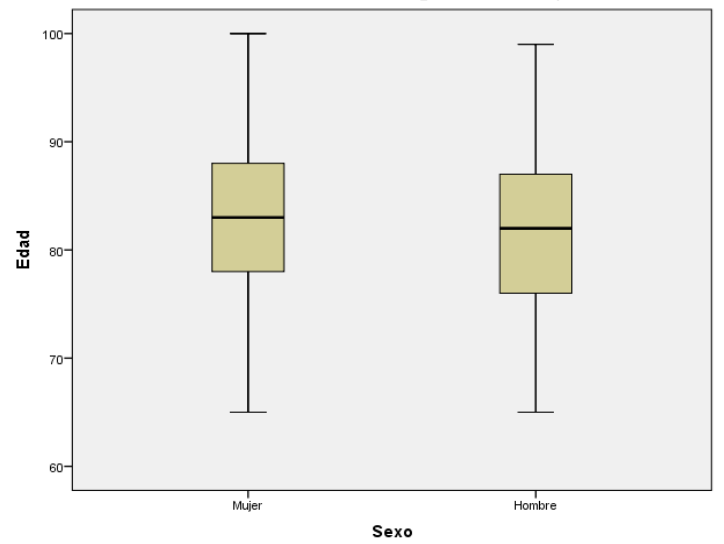

\section{Instrumentos}

Se desarrolló una ficha con recogida de datos socio demográficos: edad, sexo y código postal, además de datos: causa de fractura, tipo de fracturas, tipo de intervención, diagnostico principal, código E, procedimientos principales, fechas de ingreso y alta de hospitalización, de ingreso y alta en unidad de ortogeriatría, motivo de alta y sesiones de fisioterapia que ha recibido el paciente durante su ingreso en hospitalización, en ortogeriatría y en la unidad de rehabilitación ambulatoria del hospital.

Estos datos han sido extraídos de la Base de Datos del Hospital, con el consentimiento del Comité Ético del Hospital Universitario de Nuestra Señora de la Candelaria.

\section{Análisis de datos}

Los datos obtenidos fueron codificados y volcados en una matriz de SPSS 20.0 (IBM Corporation, 2013). Para el análisis de los datos se ha trabajo con las variables numéricas de estadística descriptiva, media y desviación estándar, mediana y percentiles intercuartílicos y frecuencias absolutas y relativas para las variables cualitativas. El contraste de hipótesis con una significación $\alpha=5 \%$, se realizan la $t$-Student y la $U$ de Mann-Whitney. 


\section{RESULTADOS}

La Unidad de Ortogeriatría comienza su andadura en el HUNSC, en febrero del 2013, por lo tanto de los datos registrados, separaremos primero los pacientes que han ingresado en la Unidad de los que no. Teniendo en cuenta que tenemos datos desde enero del 2012.

Del total de la muestra 376 pacientes: un 40\% (146) han sido atendidos en esta Unidad y 230 no.

Analizando las sesiones de fisioterapia ambulatoria totales que recibieron los pacientes, comparando entre los pacientes que estuvieron ingresados o no en la unidad de ortogeriatría podemos comprobar (Figura 2):

Figura 2. Distribución de las sesiones de fisioterapia ambulatoria r/c lugar de estancia hospitalaria

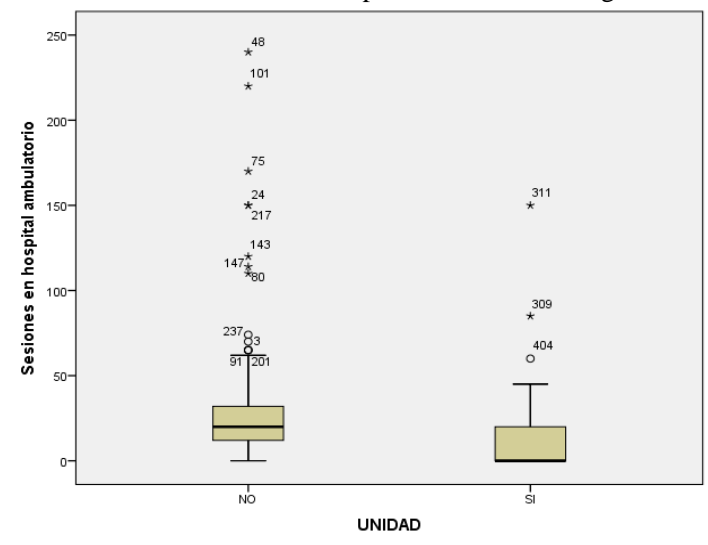

Cuando no había unidad de Ortogeriatría la media de sesiones era de 27.03, pero con mínimo de 0 y un máximo de 240 , la distribución no se ve homogénea por lo que es más apropiado hacer una valoración de percentiles.

Tenemos un percentil 50 de 20 sesiones y un recorrido intercuartílico entre de 12 y 32 ( $P=50[12-32]$ ) (Figura 3$)$.

Esto nos indica que de los paciente ancianos intervenidos con fractura de cadera que no han sido hospitalizados en ortogeriatría el $50 \%$ de ellos han tenido menos de 20 sesiones de fisioterapia ambulatoria y un $75 \%$ ha tenido menos de 32 sesiones.

Si observamos la muestra de pacientes que si han sido ingresados en la unidad de Ortogeriatría, los percentiles de distribuyen $P=0[0-20]$, lo que nos indica que los pacientes ancianos intervenidos de cadera que han sido trasladados del hospital convencional a la Unidad de Ortogeriatría, no han necesitado acudir a recibir sesiones de fisioterapia ambulatoria el 50\% de los pacientes de la muestra. 
Figura 3. Prueba U-de Mann Whitney de muestras independientes, sobre las sesiones de fisioterapia ambulatoria

UNIDAD

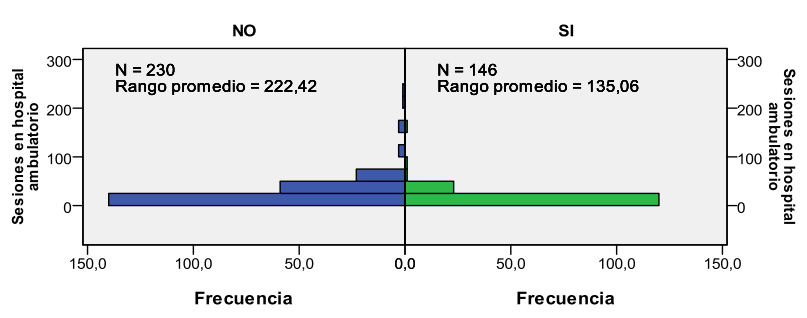

\begin{tabular}{|lr|}
\hline N total & 376 \\
\hline U de Mann-Whitney & $8.987,500$ \\
\hline W de Wilcoxon & $19.718,500$ \\
\hline Probar estadística & $8.987,500$ \\
\hline Error tipico & $1.000,829$ \\
\hline $\begin{array}{l}\text { Estadistica de prueba } \\
\text { estandarizada }\end{array}$ & $-7,796$ \\
\hline $\begin{array}{l}\text { Sig. asintótica (prueba de dos } \\
\text { caras) }\end{array}$ &, 000 \\
\hline
\end{tabular}

\section{CONCLUSIÓN}

"Las Unidades de Ortogeriatría permiten reducir el 30\% el tiempo de recuperación en el anciano con fractura de cadera", como expuso Bermejo (2013) en las I Jornadas clínico-gestoras de Ortogeriatría en la Comunidad de Madrid.

El trabajo multidisciplinar, la coordinación y creación de protocolos en los pacientes ancianos, que tienen una patología tan frecuente como la fractura de cadera, y que puede llegar a complicarse mucho, debido a las patologías concomitantes que estos pacientes pueden presentar como; hipertensión, diabetes, demencia, cardiopatías..., y las posible complicaciones (úlceras por presión, demencia, desequilibrios electrolíticos...) hace necesario una actuación rápida y coordinada que permita devolverlos a su entorno en las mejores condiciones posibles (González, Alarcón, Pallardo, Gotor, Mauleón y Gil, 2008; Ortiz, Vidán, Álvarez, García, Alonso y Serra, 2008).

El estudio realizado en el HUNSC en el corto periodo que lleva en funcionamiento el protocolo de pacientes ancianos con fractura de cadera y su integración en la unidad de ortogeriatría que contempla estancias de hospitalización y su derivación al Hospital de Ofra, para continuar tratamiento y estabilizar el paciente, ha permitido analizar, desde el área de la rehabilitación, que el inicio precoz de la atención por el fisioterapeuta, desde la hospitalización el mismo día de la intervención y el 
entrenamiento y formación al paciente y a sus familiares, las movilizaciones adecuadas, el alivio del dolor y las posturas correctas en la cama, en sedestación y en el inicio de la deambulación con apoyo, de andadora o de muletas ha permitido que cuando el paciente se ha ido de alta y tras la revisión del médico rehabilitador y la valoración de su estado funcional, no ha sido necesario continuar el tratamiento de fisioterapia ambulatoria, pues han conseguido, en su mayoría, una independencia suficiente para adaptarse a su entorno, su vida familiar y a su comunidad de forma adecuada.

Futuras investigaciones deberían contemplar valoración de costes (De la Torre et al., 2012; Juste, 2012), que nos indicarían si existe un ahorro económico añadido al beneficio que supone el no tener que trasladar a un paciente anciano en trasporte sanitario a una unidad de rehabilitación ambulatoria, para poder conseguir cierta independencia y adaptación a su vida cotidiana.

\section{REFERENCIAS}

Bermejo, R. (2013, mayo). Las unidades de Ortogeriatría permiten reducir el 30\% el tiempo de recuperación en el anciano con fractura de cadera Ponencia Artículo procedente de Sedisa Siglo XXI, I Jornada clínico-gestora "Ortogeriatría en la Comunidad de Madrid.

Bielza, R., Ortiz, A., Arias, E., Velasco, R., Mora, A., Moreno, R., Tapia, B., Escalera, J. y Gómez, J. (2013). Implantación de una Unidad de Ortogeriatría de Agudos en un hospital de segundo nivel. Revista Española de Geriatría y Gerontología, 48(1), 26-29.

Binder, E.F., Brown, M., Sinacore, D.R., Steger-May, K., Yarasheski, K.E. y Schechtman, K.B. (2004). Effects of extended outpatient rehabilitation after hip fracture: a randomized controlled trial. JAMA, 292(7), 837-46.

Comisión Europea (2003). Plan de acción. Informe sobre los próximos pasos clave para conseguir una Europa sin fracturas por fragilidad. Grupo de Consultores sobre Osteoporosis en la Comunidad Europea.

Crotty, M., Enroje, K.D., Cameron, I., Miller, M., Ramírez, G. y Couzner, L. (2010). Intervenciones de rehabilitación para mejorar el funcionamiento físico y psicosocial después de la fractura de cadera en pacientes de edad avanzada (Revisión Cochrane traducida). Biblioteca Cochrane Plus (1).

De la Torre, M., Rodríguez, J.C., Moreno, N., Jacinto, R.L., Hernández, A. y Deive, J.C. (2012). Estudio del impacto económico de las fracturas de cadera en nuestro medio. (Norte Gran Canaria). Trauma Fundación MAPFRE, 23(1), 15-21.

Instituto Nacional de Estadística (2013). [En línea] consultado: 28 de marzo 2014.

Ministerio de Sanidad y Política Social (2010). Instituto de Información Sanitaria. Estadísticas Comentadas: La Atención a la Fractura de Cadera en los Hospitales del SNS.

Ministerio de Sanidad, Servicios Sociales e Igualdad (2012). Instituto de Información Sanitaria. La hospitalización en el Sistema Nacional de Salud CMBD-Registro de altas. Informe resumen 2010 [en línea].

Ministerio de Sanidad, Política Social e Igualdad (2010). Guía de práctica clínica sobre osteoporosis y prevención de fracturas por fragilidad.

Europa Press (2009, 6 noviembre). La Unidad de Ortogeriatría del Hospital La Paz acelera la recuperación de los ancianos con fractura de cadera. 
Ganeglius, Y. (2011). Evaluación de la marcha en el adulto mayor. Carta Geriátrico Gerontológica, 4(1), 1-36.

González, J., Alarcón, T., Pallardo, B., Gotor, P., Mauleón, J. y Gil, E. (2008). Ortogeriatría en pacientes agudos (I). Aspectos asistenciales. Rev Esp Geriatr Gerontol, 43(4), 239-51.

Grupo de Estudio e Investigación de la Osteoporosis de la Sociedad Española de Cirugía Ortopédica y Traumatología, GEIOS (2011). Tratamiento multidisciplinar de las fracturas de cadera.

Guías Clínicas de la Sociedad Gallega de Medicina Interna (2005). Guía clínica para la atención de los pacientes con fractura de cadera.

Handoll, H.H., Sherrington, C. y Parker, M.J. (2004). Mobilization strategies after hip fracture surgery in adults. Cohrane Database Syst Rev, 4.

Handoll, H.H.G. y Sherrington, C. (2007). Estrategias de movilización después de la cirugía por fractura de cadera en adultos (Cochrane Review). La Biblioteca Cochrane Plus, 2.

Hernlund, E., Svedbom, A., Ivergard, M., Compston, J., Cooper, C.J., Stenmark, J. et al. (2013). Osteoporosis in the European Union: medical management, epidemiology and economic burden. A report prepared in collaboration with the International Osteoporosis Foundation (IOF) and the European Federation of Pharmaceutical Industry Associations (EFPIA). Arch Osteoporos, 8(1-2), 136.

Hoeing, H., Sanford, J., Butterfield, T., Griffiths, P., Richardson, P. y Hargraves, K. (2006). Development of a tele technology protocol for in-home rehabilitation. $J$ Rehabil Res Dev. (JRRD), 43(2), 287-98.

Hospital de Donostia Protocolo (2007). Protocolo de Fracturas de Cadera.

Hospital La Fuentefría Comunidad de Madrid (2005). Vía Clínica de Rehabilitación de cadera.

IBM Corp. Released (2013). IBM SPSS Statistics for windows, Version 20.0 Armonk, NY: IBM Corp.

Juste, M. (2012). Morbimortalidad asociada a la fractura de cadera del anciano. Análisis de nuestro medio. Trabajo de Investigación para doctorado, Departamento de Cirugía Universidad Autónoma de Barcelona,

Liu, C. y Latham, N.K. (2009). Entrenamiento de fuerza con resistencia progresiva para mejorar la función física en adultos mayores (Revisión Cochrane traducida). Biblioteca Cochrane Plus, 3.

Mangione, K., Craik, R., Tomlinson, S. y Palombaro, K. (2005). Can elderly patients who have had a hip fracture perform moderate- to high-intensity exercise at home? 'Physical Therapy, 85(8), 727-39.

Minns, C.J., Barker, K.L., Dewey, M.E. y Sackley, C.M. (2009). Effectiveness of physiotherapy exercise following hip arthroplasty for osteoarthritis: a systematic review of clinical trials. BMC Musculoskeletal Disorders, 10, 98.

Moseley, A.M., Sherrington, C., Lord, S.R., Barraclough, E., St George, R.J. y Cameron, I.D. (2009). 'Mobility training after hip fracture: a randomised controlled trial' Age Ageing, 38(1), 74-80.

National Clinical Guideline Centre (2011).'The management of hip fracture in Adults'. NICE National Clinical Guideline Centre

Olias de Lima, I. (2012). 'Vía Clínica de Fracturas en las Extremidades en Urgencias y Emergencias'. Reduca (Enfermería, Fisioterapia y Podología) Serie Trabajos de Fin de Grado, 4(1), 195-232.

Ortiz, F.J., Vidán, M., Marañón, E., Álvarez, L., García, M.A., Alonso, M., Toledano, M. y Serra, J.A. (2008). Evolución prospectiva de un programa de intervención geriátrica interdisciplinaria y secuencial en la recuperación funcional del anciano con fractura de cadera. Trauma Fundación MAPFRE, 19(1), 13-21. 
Parker, M. (2006). Hip fracture. BMJ British Medical Journal, 333, 27-30.

Pidemunt, G. (2009). Factores determinantes del deterioro de la función y calidad de vida del anciano afecto con fractura de cadera. Proyecto para optar título doctorado. Doctora en Medicina y Cirugía, Universidad Autónoma de Barcelona.

Protocolo de Actuación en las Fracturas de Cadera en el anciano en el Hospital General Universitario de Alicante (2007).

Sánchez-Lacuesta, J. (1993). Biomecánica de la marcha humana normal y patológica. Instituto de Biomecánica de Valencia. Martín Impresores, SL.

Serra, J.A., Garrido, G., Vidan, M., Marañón. E., Brañas, F. y Ortiz, J. (2002). Epidemiología de la fractura de cadera en ancianos en España. An Med Interna, 19, 389-95.

Sociedad Española de Geriatría y Gerontología (SEGG, 2007). Guía de buena práctica en geriatría. Anciano afecto de fractura de cadera [en línea]. Editorial Elsevier Doyma.

Tinetti, M.E., Baker, D.I., Gottschalk, M., Williams, C.S., Pollack, D. y Garrett, P. (1999). Homebased multicomponent rehabilitation program for persons after hip fracture: a randomized trial. Archives of Physical Medicine and Rehabilitation, 80(8), 916-22.

Recibido: 25 de abril de 2014 Recepción Modificaciones: 26 de mayo de 2014

Aceptado: 14 de junio de 2014 